\title{
A CHARACTERIZATION OF EPI-CONVERGENCE IN TERMS OF CONVERGENCE OF LEVEL SETS
}

\author{
GERALD BEER, R. T. ROCKAFELLAR, AND ROGER J.-B. WETS
}

(Communicated by Andrew M. Bruckner)

\begin{abstract}
Let $\operatorname{LSC}(X)$ denote the extended real-valued lower semicontinuous functions on a separable metrizable space $X$. We show that a sequence $\left\langle f_{n}\right\rangle$ in $\operatorname{LSC}(X)$ is epi-convergent to $f \in \operatorname{LSC}(X)$ if and only for each real $\alpha$, the level set of height $\alpha$ of $f$ can be recovered as the Painlevé-Kuratowski limit of an appropriately chosen sequence of level sets of the $f_{n}$ at heights $\alpha_{n}$ approaching $\alpha$. Assuming the continuum hypothesis, this result fails without separability. An analogous result holds for weakly lower semicontinuous functions defined on a separable Banach space with respect to Mosco epi-convergence.
\end{abstract}

\section{INTRODUCTION}

An extended real-valued function $f: X \rightarrow[-\infty, \infty]$ on a metrizable space $X$ is called lower semicontinuous provided its epigraph

$$
\text { epi } f \equiv\{(x, \alpha): x \in X, \alpha \in R \text {, and } \alpha \geq f(x)\}
$$

is a closed subset of $X \times R$. Alternatively, $f$ is lower semicontinuous provided for each real $\alpha$, the level set at height $\alpha$ of $f, \operatorname{lev}(f, \alpha) \equiv\{x \in X: f(x) \leq \alpha\}$, is a closed subset of $X$. A fundamental convergence concept for sequences of lower semicontinuous functions in optimization theory, decision theory, homogenization problems, the theory of integral functionals, algorithmic procedures, and variational analysis is the notion of epi-convergence (see, e.g., [MB, W1, W2, A, DG, V, RW, BL1, AF, BDM]). Given a sequence $\left\langle f_{n}\right\rangle$ of lower semicontinuous functions on a metric space $\langle X, d\rangle$, we say that $\left\langle f_{n}\right\rangle$ is epi-convergent to $f$, and we write $f=e-\lim f_{n}$, provided at each $x \in X$, the following two conditions both hold:

(1) whenever $\left\langle x_{n}\right\rangle$ is convergent to $x$, we have $f(x) \leq \liminf f_{n}\left(x_{n}\right)$;

(2) there exists a sequence $\left\langle x_{n}\right\rangle$ convergent to $x$ such that $f(x)=$ $\lim f_{n}\left(x_{n}\right)$.

Received by the editors April 3, 1991.

1991 Mathematics Subject Classification. Primary 49J45, 54B20, 54C35.

Key words and phrases. Epi-convergence, level sets, lower semicontinuous function, PainlevéKuratowski convergence, Mosco convergence.

The research of the first author was partially supported by NSF grant DMS-9001096. The research of the second author was partially supported by NSF grant DMS-8819586. The research of the third author was partially supported by NSF grant DMS-8922396. 
Epi-convergence of $\left\langle f_{n}\right\rangle$ of $f$ amounts to the Painlevé-Kuratowski convergence [K, §29] of the sequence of epigraphs $\left\langle\right.$ epi $\left.f_{n}\right\rangle$ to epi $f$ [A, Theorem 1.39]. Recall that given closed but possibly empty subsets $A, A_{1}, A_{2}, A_{3}, \ldots$ of $X$, $\left\langle A_{n}\right\rangle$ is declared Painlevé-Kuratowski convergent to $A$ provided $A=\operatorname{Li} A_{n}=$ Ls $A_{n}$, where

Li $A_{n}=\left\{x \in X:\right.$ there exists a sequence $\left\langle a_{n}\right\rangle$ convergent to $x$ with $a_{n} \in A_{n}$ for all but finitely many integers $\left.n\right\}$,

Ls $A_{n}=\left\{x \in X\right.$ : there exist positive integers $n_{1}<n_{2}<n_{3}<\cdots$ and $a_{k} \in A_{n_{k}}$ such that $\left.\left\langle a_{k}\right\rangle \rightarrow x\right\}$.

When $A=\operatorname{Li} A_{n}=\mathrm{Ls} A_{n}$, we write $A=\mathrm{PK}-\lim A_{n}$. Thus, $\left\langle f_{n}\right\rangle$ is epiconvergent to $f$ if and only if epi $f=\mathrm{PK}-\lim$ epi $f_{n}$. As is well-known, even for convex functions, epi-convergence is neither stronger nor weaker than pointwise convergence [SW, W1].

The main result of this note asserts that for a separable metrizable space $X$, if $f, f_{1}, f_{2}, f_{3}, \ldots$ are lower semicontinuous functions on $X$ and $\left\langle f_{n}\right\rangle$ is epi-convergent to $f$, then for each real number $\alpha, \operatorname{lev}(f, \alpha)$ can be recovered as the Painlevé-Kuratowski limit of some sequence of level sets $\left\langle\operatorname{lev}\left(f_{n}, \alpha_{n}\right)\right\rangle$ where $\left\langle\alpha_{n}\right\rangle \rightarrow \alpha$. Furthermore, this level set convergence property actually characterizes epi-convergence. Finally, we show that this result necessarily fails without separability of $X$, assuming the continuum hypothesis.

\section{SOME BACKGROUND MATERIAL}

Unless otherwise specified, $X$ will denote a metrizable space with an underlying metric $d$. When working with epigraphs, one must consider the product space $X \times R$, and in this space, the box metric will be understood: $\rho\left[\left(x_{1}, \alpha_{1}\right),\left(x_{2}, \alpha_{2}\right)\right]=\max \left\{d\left(x_{1}, x_{2}\right),\left|\alpha_{1}-\alpha_{2}\right|\right\}$. For $a \in X$ and $\varepsilon>0$, we write $S_{\varepsilon}[a]$ for the open ball with center $a$ and radius $\varepsilon$. For $A \subset X$ nonempty and $x \in X, d(x, A)=\inf \{d(x, a): a \in A\}$, and we adopt the convention $d(x, \varnothing)=\infty$. The e-enlargement of a subset $A$ of $X$ is the set

$$
S_{\varepsilon}[A] \equiv \bigcup_{a \in A} S_{\varepsilon}[a]=\{x \in X: d(x, A)<\varepsilon\} .
$$

In any metrizable space $X$ (more generally in any first countable space), Painlevé-Kuratowski convergence of sequences of closed sets is compatible with the Fell topology [Fe], also called the topology of closed convergence, on the space of all closed subsets $2^{X}$ of $X$ (see, e.g., [FLL, p. 353] or [Bel, Lemma 1.0]). To introduce this topology, we need some notation. For $E$ a nonempty subset of $X$, we designate the following subsets of $2^{X}$ :

$$
E^{-} \equiv\left\{A \in 2^{X}: A \cap E \neq \varnothing\right\}, \quad E^{+} \equiv\left\{A \in 2^{X}: A \subset E\right\} .
$$

With this notation in mind, the Fell topology $\tau_{F}$ on $2^{X}$ has as a subbase all sets of the form $V^{-}$and $V$ is an open subset of $X$, and all sets of the form $\left(K^{c}\right)^{+}$where $K$ is a compact subset of $X$. Notice that $A \in V^{-}$means that $A$ hits the open set $V$, whereas $A \in\left(K^{c}\right)^{+}$means that $A$ misses the compact set $K$. By the compatibility of Painlevé-Kuratowski convergence with the Fell 
topology, we mean precisely this: given closed subsets $A, A_{1}, A_{2}, \ldots$ of $X$, $A \subset \operatorname{Li} A_{n}$ if and only if for each open subset $V$ of $X$ with $A \cap V \neq \varnothing$, we have $A_{n} \cap V \neq \varnothing$ eventually, and Ls $A_{n} \subset A$ if and only if for each compact subset $K$ of $X$ with $A \cap K=\varnothing$, we have $A_{n} \cap K=\varnothing$ eventually.

It turns out that $\tau_{F}$ is Hausdorff if and only if $X$ is locally compact and that with no assumptions whatsoever on $X$, the hyperspace $\left\langle 2^{X}, \tau_{F}\right\rangle$ is compact [A, p. 251]! If $X$ is separable, then the topology is sequentially compact, i.e., each sequence $\left\langle A_{n}\right\rangle$ in $2^{X}$ has a subsequence that is Painlevé-Kuratowski convergent to some closed set $A$ (see, e.g., [K, p. 340] or [AF, p. 23]). If $X$ is both locally compact and separable, then $\tau_{F}$ is metrizable as well as compact [A, Proposition 2.77].

$\operatorname{LSC}(X)$ will represent the lower semicontinuous functions with domain $X$ and values in $[-\infty, \infty]$. For $f \in \operatorname{LSC}(X)$, we denote $\inf \{f(x): x \in X\}$ by $v(f)$. We call $f$ proper provided that $f$ is somewhere finite and for each $x$, we have $f(x)>-\infty$. For $f$ proper and lower semicontinuous, the possibly empty closed subset of minimizers of $f$ will be denoted by $\operatorname{Argmin} f$. The following facts about level sets and the value function $f \rightarrow v(f)$ are known:

(a) if $f=e-\lim f_{n}$, then $v(f) \geq \limsup v\left(f_{n}\right)$ [A, Proposition 2.9];

(b) if $f=e-\lim f_{n}$ and $\left\langle\alpha_{n}\right\rangle \rightarrow \alpha$, the $\operatorname{lev}(f, \alpha) \supset \operatorname{Ls} \operatorname{lev}\left(f_{n}, \alpha_{n}\right)[\mathrm{V}$, p. 199; W2, Theorem 1].

(c) if $f=e-\lim f_{n}$, and if each $f_{n}$ is convex, then $f$ is convex and for each $\alpha>v(f)$, we have $\operatorname{lev}(f, \alpha)=\mathrm{PK}-\lim \operatorname{lev}\left(f_{n}, \alpha\right)$ [Mo2, Lemma 3.1];

(d) if $f=e-\lim f_{n}$ and $v(f)=\lim v\left(f_{n}\right)$ and for each $\alpha>v(f)$ we have $\operatorname{lev}(f, \alpha)=\operatorname{cl}\{x: f(x)<\alpha\}$, then $f=e-\lim f_{n}$ if and only for each $\alpha>v(f)$, we have $\operatorname{lev}(f, \alpha)=\mathrm{PK}-\lim \operatorname{lev}\left(f_{n}, \alpha\right)$ [St, Théoreme 2.1; BL1, Theorem 5.1].

To appreciate properly our characterization of epi-convergence, some counterexamples are in order. First in the convex case, it is clear that $f=e-\lim f_{n}$ need not force Argmin $f$ to agree with PK-lim Argmin $f_{n}$, even if $\operatorname{Argmin} f_{n}$ is nonempty and $v(f)=\lim v\left(f_{n}\right)$ for each $n$. On the line, let $f, f_{1}, f_{2}, f_{3}, \ldots$ be defined by

$$
\begin{gathered}
f(x)= \begin{cases}0 & \text { if } x \geq 0 \\
\infty & \text { if } x<0\end{cases} \\
f_{n}(x)=\left\{\begin{array}{ll}
\left(1 / n^{2}\right)|x-n| & \text { if } x \geq 0, \\
\infty & \text { if } x<0,
\end{array} \text { for } n=1,2,3, \ldots .\right.
\end{gathered}
$$

On the other hand, in the convex case, $\operatorname{lev}(f, \alpha)=\mathrm{PK}-\lim \operatorname{lev}\left(f_{n}, \alpha\right)$ for each $\alpha \geq v(f)$ need not force $f=e-\lim f_{n}$ : on the line, take $f \equiv 0$ and $f_{n} \equiv-n$.

For nonconvex but quasi-convex functions, $f=e-\lim f_{n}$ does not guarantee convergence of level sets at heights above $v(f)$ : Let $f, f_{1}, f_{2}, f_{3}, \ldots$ be defined by

$$
\begin{gathered}
f(x)= \begin{cases}0 & \text { if } x=0, \\
1 & \text { if } x \neq 0\end{cases} \\
f_{n}(x)=\left\{\begin{array}{ll}
0 & \text { if } x=0, \\
1+1 / n & \text { if } x \neq 0,
\end{array} \text { for } n=1,2,3, \ldots\right.
\end{gathered}
$$

Then $\operatorname{lev}(f, 1) \neq \mathrm{PK}-\lim \operatorname{lev}\left(f_{n}, 1\right)$. 


\section{The MAIN RESUlT}

Theorem 3.1. Let $X$ be a separable metrizable space and let $f, f_{1}, f_{2}, \ldots$ be extended real valued lower semicontinuous functions on $X$.

(1) If $f=e-\lim f_{n}$, then for each $\alpha \in R$ there exists a sequence $\left\langle\alpha_{n}\right\rangle$ of reals convergent to $\alpha$ such that $\operatorname{lev}(f, \alpha)=\mathrm{PK}-\lim \operatorname{lev}\left(f_{n}, \alpha_{n}\right)$;

(2) if for each $\alpha \in R$ there exists a sequence $\left\langle\alpha_{n}\right\rangle$ of reals convergent to $\alpha$ with $\operatorname{lev}(f, \alpha)=\mathrm{PK}-\lim \operatorname{lev}\left(f_{n}, \alpha_{n}\right)$, then $f=e-\lim f_{n}$.

Proof of Theorem 3.1. (1) Fix $\alpha \in R$. As mentioned in $\S 2$, it is well known that for any sequence $\left\langle\alpha_{n}\right\rangle$ convergent to $\alpha$, we have $\operatorname{Ls} \operatorname{lev}\left(f_{n}, \alpha_{n}\right) \subset \operatorname{lev}(f, \alpha)$. For completeness, we supply a proof. Suppose $x \in \operatorname{Ls} \operatorname{lev}\left(f_{n}, \alpha_{n}\right)$, i.e., there exist indices $n_{1}<n_{2}<n_{3}<\cdots$ and $x_{n_{k}} \in \operatorname{lev}\left(f_{n_{k}}, \alpha_{n_{k}}\right)$ for $k=1,2,3, \ldots$ such that $\left\langle x_{n_{k}}\right\rangle \rightarrow x$. For $n \notin\left\{n_{k}: k \in Z^{+}\right\}$, set $x_{n}=x$. Then $\left\langle x_{n}\right\rangle \rightarrow x$; so, by epi-convergence, we have

$$
f(x) \leq \liminf _{n \rightarrow \infty} f_{n}\left(x_{n}\right) \leq \liminf _{k \rightarrow \infty} f_{n_{k}}\left(x_{n_{k}}\right) \leq \liminf _{k \rightarrow \infty} \alpha_{n_{k}}=\alpha .
$$

This proves that $x \in \operatorname{lev}(f, \alpha)$.

To establish the companion inclusion $\operatorname{lev}(f, \alpha) \subset \operatorname{Lilev}\left(f_{n}, \alpha_{n}\right)$ requires separability and a judicious choice of the scalars $\left\langle\alpha_{n}\right\rangle$. Let $\left\langle x_{i}\right\rangle$ be a sequence in $\operatorname{lev}(f, \alpha)$ whose set of cluster points is $\operatorname{lev}(f, \alpha)$ (this is a stronger requirement than density of $\left\{x_{i}: i \in Z^{+}\right\}$in $\left.\operatorname{lev}(f, \alpha)\right)$. Since epi $f \subset \operatorname{Li}$ epi $f_{n}$, for each positive integer $m$, there exists a positive integer $N_{m}$ such that $\forall n \geq N_{m}$ there exists points $\left\{\left(w_{i n}^{(m)}, \alpha_{i n}^{(m)}\right): i=1,2,3, \ldots, m\right\}$ in epi $f_{n}$ such that for each $i \in\{1,2, \ldots, m\}$, we have both $d\left(w_{i n}^{(m)}, x_{i}\right)<1 / m$ and $\left|\alpha_{i n}^{(m)}-\alpha\right|<1 / m$. Without loss of generality, we may assume that $\left\langle N_{m}\right\rangle$ is a strictly increasing sequence. We now may define our sequence of scalars $\left\langle\alpha_{n}\right\rangle$ : take $\alpha_{n}=\alpha+1$ for $n<N_{1}$ and for $N_{m} \leq n<N_{m+1}$, take $\alpha_{n}=\alpha+1 / m, m=1,2,3,4, \ldots$. We now show that this choice does the job.

To show that $\operatorname{lev}(f, \alpha) \subset \operatorname{Lilev}\left(f_{n}, \alpha_{n}\right)$, we find it convenient to work with the Fell topology equivalent as described in $\S 2$. To this end, suppose $\operatorname{lev}(f, \alpha) \cap$ $V \neq \varnothing$ where $V$ is an open subset of $X$. Pick $x \in \operatorname{lev}(f, \alpha) \cap V$ and $\varepsilon>0$ such that $S_{\varepsilon}[x] \subset V$. Since $x$ is a cluster point of $\left\langle x_{i}\right\rangle$, we may choose $k \in Z^{+}$so that both $1 / k<\varepsilon / 2$ and $d\left(x_{k}, x\right)<\varepsilon / 2$. Fix $n \geq N_{k}$; then there is a largest integer $m$ such that $n \geq N_{m}$. We have $d\left(w_{k n}^{(m)}, x_{k}\right)<1 / m$ and $\left|\alpha_{k n}^{(m)}-\alpha\right|<1 / m$. Since $f_{n}\left(w_{k n}^{(m)}\right) \leq \alpha_{k n}^{(m)}<\alpha+1 / m=\alpha_{n}$, we have $w_{k n}^{(m)} \in \operatorname{lev}\left(f_{n}, \alpha_{n}\right)$. Also,

$$
d\left(w_{k n}^{(m)}, x\right) \leq d\left(w_{k n}^{(m)}, x_{k}\right)+d\left(x_{k}, x\right)<\frac{1}{m}+\frac{\varepsilon}{2} \leq \frac{1}{k}+\frac{\varepsilon}{2}<\varepsilon,
$$

so that $w_{k n}^{(m)} \in V$. This means that for each $n \geq N_{k}$, we have $V \cap \operatorname{lev}\left(f_{n}, \alpha_{n}\right) \neq$ $\varnothing$. We may now assert that $\operatorname{lev}(f, \alpha) \subset \operatorname{Lilev}\left(f_{n}, \alpha_{n}\right)$, so that $\operatorname{lev}(f, \alpha)=$ PK $-\lim \operatorname{lev}\left(f_{n}, \alpha_{n}\right)$.

(2) The condition $\operatorname{lev}(f, \alpha) \subset \operatorname{Lilev}\left(f_{n}, \alpha_{n}\right)$, valid for each $\alpha \in R$ and for some sequence $\left\langle\alpha_{n}\right\rangle$ convergent to $\alpha$, immediately implies that epi $f \subset$ Li epi $f_{n}$. In fact, if we just know that $\operatorname{lev}(f, \alpha) \subset \operatorname{Lilev}\left(f_{n}, \alpha_{n}\right)$ for each $\alpha>v(f)$, then epi $f \subset$ Li epi $f_{n}$ holds. The details are left to the reader. 
To see that Ls epi $f_{n} \subset$ epi $f$, suppose to the contrary that $(x, \beta) \in$ Ls epi $f_{n}$ but that $(x, \beta) \notin$ epi $f$. Then $\beta<f(x)$. We can find an increasing sequence of integers $n_{1}<n_{2}<n_{3}<n_{4}<\ldots$ and $\left(x_{k}, \beta_{k}\right) \in$ epi $f_{n_{k}}$ such that $\left\langle\left(x_{k}, \beta_{k}\right)\right\rangle$ is convergent to $(x, \beta)$. Choose a scalar $\alpha$ between $\beta$ and $f(x)$, and let $\left\langle\alpha_{n}\right\rangle$ be a sequence of scalars convergent to $\alpha$ for which $\operatorname{lev}(f, \alpha)=$ PK $-\lim \operatorname{lev}\left(f_{n}, \alpha_{n}\right)$. Then for all $k$ sufficiently large, we have $\beta_{k}<\alpha_{n_{k}}$. For all such $k$, we have $x_{k} \in \operatorname{lev}\left(f_{n_{k}}, \alpha_{n_{k}}\right)$ and the condition $\operatorname{Ls} \operatorname{lev}\left(f_{n}, \alpha_{n}\right) \subset$ $\operatorname{lev}(f, \alpha)$ now yields $x \in \operatorname{lev}(f, \alpha)$. This contradicts $f(x)>\alpha$.

The proof of the second assertion in the statement of Theorem 3.1 does not use separability of $X$. On the other hand, if we accept the continuum hypothesis, the first assertion fails in each nonseparable space.

Example. Let $\langle X, d\rangle$ be a nonseparable metric space. By Zorn's Lemma, for each $\varepsilon>0$ we can find a maximal $\varepsilon$-discrete subset $W_{\varepsilon}$ of $X$, i.e., for each $w_{1}$ and $w_{2}$ in $W_{\varepsilon}$, we have $d\left(w_{1}, w_{2}\right) \geq \varepsilon$. By the maximality of $W_{\varepsilon}$, we have $S_{\varepsilon}\left[W_{\varepsilon}\right]=X$. Thus, by nonseparability, there exists some $\varepsilon_{0}>0$ for which $W \equiv W_{\varepsilon_{0}}$ is uncountable. Let $\Omega$ be the following set of rational sequences:

$$
\Omega=\left\{g: Z^{+} \rightarrow Q: \forall k \in Z^{+}, g(k)>0, \text { and } \lim _{k \rightarrow \infty} g(k)=0\right\} .
$$

Then $\Omega$ has the cardinality of the continuum; so, by the continuum hypothesis, there exists a one-to-one function $\varphi: \Omega \rightarrow W$. Let $f: X \rightarrow R$ be the characteristic function of $\varphi(\Omega)^{c}$. We produce a sequence $\left\langle f_{n}\right\rangle$ of real-valued lower semicontinuous functions epi-convergent to $f$ for which condition (1) in the statement of Theorem 3.1 fails.

For each $g \in \Omega$, let us write $w_{g}$ for $\varphi(g)$. We define $f_{n}: X \rightarrow R$ by

$$
f_{n}(x)= \begin{cases}g(n) & \text { if } x=w_{g} \text { for some } g \\ 1 & \text { otherwise }\end{cases}
$$

Clearly $\left\langle f_{n}\right\rangle$ is pointwise and epi-convergent to $f$. Let $\left\langle\alpha_{n}\right\rangle$ be an arbitrary sequence of reals tending to zero. We show that

$$
\varphi(\Omega)=\operatorname{Argmin} f=\operatorname{lev}(f, 0) \not \subset \operatorname{Lilev}\left(f_{n}, \alpha_{n}\right) .
$$

First, without loss of generality, we may assume that $\alpha_{n}>0$ for each $n$, for replacing $\alpha_{n}$ by $\max \left\{\alpha_{n}, 1 / n\right\}$ results in a larger level set for each $n$. Now choose $g \in \Omega$ such that for each $n$ we have $\alpha_{n}<g(n)<2 \alpha_{n}$. We claim that although $w_{g} \in \operatorname{lev}(f, 0)$, we have $w_{g} \notin \operatorname{Lilev}\left(f_{n}, \alpha_{n}\right)$. To see this, notice that $S_{\varepsilon_{0}}\left(w_{g}\right)$ contains no other points of $W$, so that for each positive integer $n$ with $g(n)<1$ we have

$$
\inf \left\{f_{n}(x): x \in S_{\varepsilon_{0}}\left(w_{g}\right)\right\}=f_{n}\left(w_{g}\right)=g(n)>\alpha_{n} .
$$

Thus, for all $n$ sufficiently large, we have $S_{\varepsilon_{0}}\left(w_{g}\right) \cap \operatorname{lev}\left(f_{n}, \alpha_{n}\right)=\varnothing$.

The general line of reasoning in our last example can be adapted to show that the sequential compactness of Painlevé-Kuratowski convergence fails without separability. Let $\Sigma$ be the set of all functions $f: Z^{+} \rightarrow\{0,1\}$ such 
that $f(n)=1$ for infinitely many $n$, and let $\psi$ be a bijection from some uncountable $\varepsilon$-discrete subset $W$ of $X$ onto $\Sigma$. For each $n \in Z^{+}$, let $A_{n}=\{x \in W: \psi(x)(n)=1\}$. Now let $\left\langle A_{n_{k}}\right\rangle$ be an arbitrary subsequence of $\left\langle A_{n}\right\rangle$. We claim that $\operatorname{Li} A_{n_{k}}$ is a proper subset of Ls $A_{n_{k}}$. To see this, choose $x \in W$ such that $\psi(x)$ is the characteristic function of $\left\{n_{2}, n_{4}, n_{6}, \cdots\right\}$. Since $x \in A_{n_{2 k}}$ for each $k$, we have $x \in \operatorname{Ls} A_{n_{k}}$. However, by the $\varepsilon$-discreteness of $W, x \notin \operatorname{Li} A_{n_{k}}$.

In metric spaces in which closed and bounded sets are compact, PainlevéKuratowski convergence of sequences of sets agrees with other convergence notions that are, in more general settings, much stronger. The most important of these is certainly convergence with respect to the metrizable bounded Hausdorff topology $\tau_{b H}$, which when specialized to epigraphs, is called the epi-distance topology [AW, ALW, AP, Be3, BL2, P]. Convergence of a sequence $\left\langle A_{n}\right\rangle$ to $A$ in this sense means uniform convergence of the associated sequence of distance functionals $\left\langle d\left(\cdot, A_{n}\right)\right\rangle$ to $d(\cdot, A)$ on bounded subsets of $X$. Alternatively, it can be shown [AP] that $\left\langle A_{n}\right\rangle$ is $\tau_{b H^{-}}$-convergent to $A$ if and only if for each nonempty bounded subset $B$ of $X$ and each $\varepsilon>0$, there exists $N \in Z^{+}$such that for each $n>N$ we have both $A \cap B \subset S_{\varepsilon}\left[A_{n}\right]$ and $A_{n} \cap B \subset S_{\varepsilon}[A]$. Notice that $\varnothing=\tau_{b H}-\lim A_{n}$ if and only if $A_{n}$ is eventually outside each bounded set. This is not the case for Painlevé-Kuratowski convergence in a general metric space.

It is indeed possible to give a shorter proof of Theorem 3.1 when closed and bounded sets are compact, using the coincidence of the two convergence notions. By virtue of the next example, we see that Theorem 3.1 remains valid when Painlevé-Kuratowski convergence is replaced by $\tau_{b H}$-convergence only in this restrictive setting.

Example. Let $\langle X, d\rangle$ be a metric space in which some closed and bounded set is noncompact, and fix $x_{0} \in X$. We produce a sequence $f, f_{1}, f_{2}, \ldots$ in $\operatorname{LSC}(X)$ with $f=\tau_{b H}-\lim f_{n}$ and with $\operatorname{Argmin} f=\left\{x_{0}\right\}$, but such that whenever $\left\langle\alpha_{n}\right\rangle \rightarrow$ $v(f)$, we do not have $\operatorname{Argmin} f=\tau_{b H}-\lim \operatorname{lev}\left(f_{n}, \alpha_{n}\right)$. Choose $\rho>0$ such that $B=\left\{x \in X: d\left(x, x_{0}\right) \leq \rho\right\}$ is noncompact. Let $x_{1}, x_{2}, x_{3}, \ldots$ be a sequence in $B$ without a cluster point, and let $\delta=\inf \left\{d\left(x_{0}, x_{k}\right): k \in Z^{+}\right\}$. We define our functions $f$ and $f_{n}$ as follows:

$$
\begin{gathered}
f(x)= \begin{cases}0 & \text { if } x=x_{0} \\
1 / k & \text { if } x=x_{k} \\
1 & \text { otherwise }\end{cases} \\
f_{n}(x)=\left\{\begin{array}{ll}
1 / n & \text { if } x=x_{0}, \\
1 / k & \text { if } x=x_{k} \text { for some } k \geq 1, \\
1 & \text { otherwise },
\end{array} \text { for } n=1,2,3, \ldots\right.
\end{gathered}
$$

Evidently, we have uniform convergence of $\left\langle f_{n}\right\rangle$ to $f$, from which $\tau_{b H}$-convergence of epigraphs follows. Clearly, Argmin $f=\left\{x_{0}\right\}$ and $v(f)=0$. Fix $n \in Z^{+}$. For the inclusion

$$
B \cap \operatorname{Argmin} f \subset S_{\delta}\left(\operatorname{lev}\left(f_{n}, \alpha_{n}\right)\right)
$$

to hold, we must have $\alpha_{n}>0$. But for each $\alpha_{n}>0, \operatorname{lev}\left(f_{n}, \alpha_{n}\right)$ must contain all $x_{k}$ for $k$ sufficiently large, so that the inclusion

$$
B \cap \operatorname{lev}\left(f_{n}, \alpha_{n}\right) \subset S_{\delta}(\operatorname{Argmin} f)
$$


must fail whenever $B \cap \operatorname{Argmin} f \subset S_{\delta}\left(\operatorname{lev}\left(f_{n}, \alpha_{n}\right)\right.$ holds. As a result, $\left\langle\operatorname{lev}\left(f_{n}, \alpha_{n}\right)\right\rangle$ cannot converge in the bounded Hausdorff topology to Argmin $f$ for any choice of $\left\langle\alpha_{n}\right\rangle$ convergent to zero.

The reader may consult [BL2,§3] for the interplay between convergence of convex functions in the epi-distance topology and the convergence of their level sets in a general normed space.

\section{THE WEAKLY LOWER SEMICONTINUOUS CASE}

In a seminal paper in the theory of set convergence, Mosco [Mol] introduced an analogue of Painlevé-Kuratowski convergence for sequences of closed convex sets in a Banach space and sequences of lower semicontinuous convex functions as identified with their epigraphs. This convergence notion has been widely studied over the past twenty years and is invariably called Mosco convergence in the literature. A sequence of closed convex sets is declared Mosco convergent $\left(A=\mathrm{M}-\lim A_{n}\right)$ to a closed convex set $A$ provided $A=\operatorname{Li} A_{n}=\operatorname{Ls} A_{n}$ with respect to the metric given by the norm, and in addition, $A=\mathrm{w}-\mathrm{Li} A_{n}=\mathrm{w}-\mathrm{Ls} A_{n}$, where $\mathrm{w}-\mathrm{Li} A_{n}$ and $\mathrm{w}-\mathrm{Ls} A_{n}$ denote the lower and upper limits of the sequence with respect to the weak topology. It is well known and easy to verify that $A=\mathrm{M}-\lim A_{n}$ if and only if $A \subset \mathrm{Li} A_{n}$ and w-Ls $A_{n} \subset A$. Mosco convergence so defined has been a particularly productive notion in the setting of reflexive spaces, for then $A=\mathrm{M}-\lim A_{n}$ implies $A^{\circ}=\mathrm{M}-\lim A_{n}^{\circ}$ and if $f=\mathrm{M}-\lim f_{n}$, i.e., epi $f=\mathrm{M}$-lim epi $f_{n}$, then $f^{*}=\mathrm{M}-\lim f_{n}^{*}$ [Mo2]. Here, $A^{\circ}$ represents the polar of a convex set $A$, and $f^{*}$ represents the conjugate of a convex function $f$, and closedness and nonemptiness of sets and properness of functions is understood. Continuity of such maps has been subsequently shown to characterize reflexivity $[\mathrm{BB}]$. We also note that in the reflexive setting, interesting connections between Mosco convergence and Banach space geometry have been revealed by several authors (see, e.g. [A, So, T, BF, BP]).

Without reflexivity, Mosco convergence of sequences of convex sets and convex functions in any Banach space is compatible with a topology of the Fell type identified in [Be2]. This topology of Mosco convergence $\tau_{M}$ on the closed convex subsets $C(X)$ of a Banach space has as a subbase all sets of the form $V^{-}$where $V$ is an open subset of $X$, and all sets of the form $\left(K^{c}\right)^{+}$where $K$ is a weakly compact subset of $X$ (this should come as no surprise). Thus, we have in any Banach space $X, A=\mathrm{M}-\lim A_{n}$ if and only if $A=\tau_{\mathrm{M}}-\lim A_{n}$ [Be2, Theorem 3.1]. Reflexivity for the Mosco topology seems to parallel local compactness for the Fell topology: $\tau_{M}$ is Hausdorff if and only if $X$ is reflexive [BB], and if $X$ is separable and reflexive then the space of nonempty closed convex subsets equipped with $\tau_{M}$ is completely metrizable [A, Be2, T].

Although it has not been frequently observed, Mosco convergence and the associated Mosco topology are also compatible for the larger class of weakly closed subsets of $X$ and for the weakly lower semicontinuous functions on $X$ (weak lower semicontinuity of $f: X \rightarrow[-\infty, \infty]$ means that epi $f$ is weakly closed in $X \times R)$. Moreover, a standard proof in the convex case [Mol, Lemma 1.10] shows that for weakly lower semicontinuous functions $f, f_{1}, f_{2}, f_{3}, \ldots$ on $X$, the relation epi $f=$ M-lim epi $f_{n}$ locally amounts to the conjunction of 
the expected two conditions: at each $x \in X$,

(1) whenever the sequence $\left\langle x_{n}\right\rangle$ is weakly convergent to $x$, we have $f(x) \leq$ $\liminf f_{n}\left(x_{n}\right)$;

(2) there exists a sequence $\left\langle x_{n}\right\rangle$ convergent strongly to $x$ such that $f(x)=$ $\lim f_{n}\left(x_{n}\right)$.

A straightforward modification of the proof of Theorem 3.1 yields this analog:

Theorem 4.1. Let $X$ be a separable Banach space and let $f, f_{1}, f_{2}, \ldots$ be extended real valued weakly lower semicontinuous functions on $X$.

(1) If $f=M-\lim f_{n}$, then for each $\alpha \in R$ there exists a sequence $\left\langle\alpha_{n}\right\rangle$ of reals convergent to $\alpha$ such that $\operatorname{lev}(f, \alpha)=M-\lim \operatorname{lev}\left(f_{n}, \alpha_{n}\right)$;

(2) if for each $\alpha \in R$ there exists a sequence $\left\langle\alpha_{n}\right\rangle$ of reals convergent to $\alpha$ with $\operatorname{lev}(f, \alpha)=M-\lim \operatorname{lev}\left(f_{n}, \alpha_{n}\right)$, then $f=M-\lim f_{n}$.

Recall that an extended real-valued function defined on a normed linear space is called quasi-convex provided its level sets are convex. As weak lower semicontinuity and lower semicontinuity for such functions coincide, we have this corollary of interest.

Corollary 4.2. Let $X$ be a separable Banach space and let $f, f_{1}, f_{2}, \ldots$ be extended real-valued lower semicontinuous quasi-convex functions on $X$.

(1) If $f=M-\lim f_{n}$, then for each $\alpha \in R$ there exists a sequence $\left\langle\alpha_{n}\right\rangle$ of reals convergent to $\alpha$ such that $\operatorname{lev}(f, \alpha)=M-\lim \operatorname{lev}\left(f_{n}, \alpha_{n}\right)$;

(2) if for each $\alpha \in R$ there exists a sequence $\left\langle\alpha_{n}\right\rangle$ of reals convergent to $\alpha$ with $\operatorname{lev}(f, \alpha)=M-\lim \operatorname{lev}\left(f_{n}, \alpha_{n}\right)$, then $f=M-\lim f_{n}$.

\section{REFERENCES}

[AF] J.-P. Aubin and H. Frankowska, Set-valued analysis, Birkhäuser, Boston, MA, 1990.

[A] H. Attouch, Variational convergence for functions and operators, Pitman, New York, 1984.

[ALW] H. Attouch, R. Lucchetti, and R. Wets, The topology of the $\rho$-Hausdorff distance, Ann. Mat. Pura Appl. (to appear).

[AW] H. Attouch and R. Wets, Quantitative stability of variational systems: I. The epigraphical distance, Trans. Amer. Math. Soc. 328 (1991), 695-730.

[AZP] D. Azé and J.-P. Penot, Operations on convergent families of sets and functions, Optimization 21 (1990), 521-534.

[BDM] J. Baxter, G. Dal Maso, and U. Mosco, Stopping times and $\Gamma$-convergence, Trans. Amer. Math. Soc. 303 (1987), 1-38.

[Be1] G. Beer, Metric spaces with nice closed balls and distance functions for closed sets, Bull. Austral. Math. Soc. 35 (1987), 81-96.

[Be2] _- On Mosco convergence of convex sets, Bull. Austral. Math. Soc. 38 (1988), 239-253.

[Be3] Conjugate convex functions and the epi-distance topology, Proc. Amer. Math. Soc. 108 (1990), 117-126.

[BB] G. Beer and J. Borwein, Mosco convergence and reflexivity, Proc. Amer. Math. Soc. 109 (1990), 427-436.

[BL1] G. Beer and R. Lucchetti, Minima of quasi-convex functions, Optimization 20 (1989), 581596.

[BL2] _ Convex optimization and the epi-distance topology, Trans. Amer. Math. Soc. 327 (1991), 795-813. 
[BP] G. Beer and D. Pai, On convergence of convex sets and relative Chebyshev centers, J. Approximation Theory 62 (1990), 147-169.

[BF] J. Borwein and S. Fitzpatrick, Mosco convergence and the Kadec property, Proc. Amer. Math. Soc. 106 (1989), 843-852.

[Fe] J. Fell, $A$ Hausdorff topology for the closed subsets of a locally compact non-Hausdorff space, Proc. Amer. Math. Soc. 13 (1962), 472-476.

[FLL] S. Francaviglia, A. Lechicki, and S. Levi, Quasi-uniformization of hyperspaces and convergence of nets of semicontinuous multifunctions, J. Math. Anal. Appl. 112 (1985), 347-370.

[DG] E. De Giorgi, Convergence problems of functionals and operators, Recent methods in nonlinear analysis (E. De Giorgi, E. Magenes and U. Mosco, eds.), Pitagora Editrice, Bologna, 1979, pp. 131-188.

[K] K. Kuratowski, Topology, vol. 1, Academic Pres, New York, 1966.

[MB] L. Mclinden and R. Bergstrom, Preservation of convergence of sets and functions in finite dimensions, Trans. Amer. Math. Soc. 268 (1981), 127-142.

[Mo1] U. Mosco, Convergence of convex sets and of solutions of variational inequalities, Adv. in Math. 3 (1969), 510-585.

[Mo2] _ On the continuity of the Young-Fenchel transform, J. Math. Anal. Appl. 35 (1971), 518-535.

[P] J.-P. Penot, The cosmic Hausdorff topology, the bounded Hausdorff topology, and continuity of polarity, Proc. Amer. Math. Soc. 113 (1991), 275-285.

[RW] R. T. Rockafellar and R. Wets, Variational systems, an introduction, in Multifunctions and integrands (G. Salinetti, ed.), Lecture Notes in Math., vol. 1091, Springer-Verlag, Berlin, 1984, pp. 1-54.

[SW] G. Salinetti and R. Wets, On the relationship between two types of convergence for convex functions, J. Math. Anal. Appl. 60 (1977), 211-226.

[So] Y. Sonntag, Convergence au sens de Mosco; théorie et applications à l'approximation des solutions d'inéquations, Thèse, Université de Provence, Marseille, 1982.

[St] M. Soueycatt, Epi-convergence et convergence des sections, Application à la stabilité des $\varepsilon$-points-selles de fonctions convexes-concaves, Publications AVAMAC, Université de Perpignan (2), 1987, 7.01-7.40.

[T] M. Tsukada, Convergence of best approximations in a smooth Banach space, J. Approximation Theory 40 (1984), 301-309.

[V] M. Volle, Contributions à la dualité en optimisation et à l'epi-convergence, Thèse, Université de Pau, Pau, France, 1986.

[W1] R. Wets, Convergence of convex functions, variational inequalities, and convex optimization problems, Variational inequalities and complementarity problems (R. Cottle, F. Gianessi, and J.-L. Lions, eds), Wiley, Chichester, 1980, pp. 375-403.

[W2] _ A formula for the level sets of epi-limits and some applications, Mathematical theories of optimization (J. Cecconi and T. Zolezzi, eds), Lecture Notes in Math., vol. 979, SpringerVerlag, Berlin, 1983, pp. 256-268.

Department of Mathematics, California State University, Los Angeles, California 90032

Department of Mathematics, University of Washington, Seattle, Washington 98195

Department of Mathematics, University of California, Davis, California 95616 